\title{
Elevación de las aminotransferasas: una nueva herramienta para el diagnóstico de coledocolitiasis. Un estudio de casos y controles
}

\section{Elevated transaminases: a new tool for the diagnosis of choledocholithiasis. A case control study}

\author{
James Yurgaky-Sarmiento, MD, ${ }^{1}$ (i) William Otero-Regino, MD,,$^{2 *}$ () Martín Gómez-Zuleta, MD. ${ }^{3}$ (1)
}

\author{
GacCeso abierto \\ Citación: \\ Yurgaky-Sarmiento J, Otero-Regino W, Gómez- \\ Zuleta M. Elevación de las aminotransferasas: \\ una nueva herramienta para el diagnóstico de \\ coledocolitiasis. Un estudio de casos y controles. \\ Rev Colomb Gastroenterol. 2020;35(3):319-328. \\ https://doi.org/10.22516/25007440.446
}

Internista, endocrinólogo, gastroenterólogo, Universidad Nacional de Colombia; Bogotá, Colombia.

2 Profesor titular de Medicina, coordinador de Gastroenterología, Universidad Nacional de Colombia, Hospital Universitario Nacional de Colombia. Gastroenterólogo, Clínica Fundadores; Bogotá, Colombia Profesor asociado de Medicina, unidad de Gastroenterología, Universidad Nacional de Colombia, Hospital Universitario Nacional de Colombia. Gastroenterólogo, Hospital de Kennedy y UGEC; Bogotá, Colombia.

*Correspondencia: William Otero-Regino, MD waoteror@gmail.com

Fecha recibido: $\quad$ 10/07/19

Fecha aceptado: 26/08/19

\begin{abstract}
Resumen
Introducción: la coledocolitiasis (CDL) afecta al $10 \%$ de los pacientes con cálculos en la vesícula biliar. La obstrucción del colédoco se asocia a pancreatitis, colangitis y ruptura del colédoco. Clásicamente, la obstrucción biliar es considerada cuando se aumentan la fosfatasa alcalina, la y-glutamil-transpeptidasa (GGTP) y las bilirrubinas. En la última década, se ha encontrado que hasta un $10 \%$ de los pacientes con CDL presentan elevaciones de las aminotransferasas. En Latinoamérica, no se ha estudiado esta alteración. El objetivo del presente trabajo fue determinar la prevalencia de la elevación de transaminasas y su evolución. Metodología: estudio de casos y controles. Se determinó la alanina aminotransferasa (ALT) al ingreso, a las $48 \mathrm{~h}$ y a las $72 \mathrm{~h}$. Si la ecografía era normal, se realizó una colangiorresonancia o una ecoendoscopia, así como una colangiopancreatografía retrógrada endoscópica (CPRE) cuando fue necesario. Resultados: se incluyeron 72 pacientes con CDL (casos) y 128 con colecistitis, sin CDL (controles). En los casos, el $83 \%$ tuvo un aumento de ALT, mientras que el 56,9\% presentó una elevación de 2-9 veces, el $16 \%$, de 10-20 veces, y el 8,3\% mostró una elevación $>20$ veces. En contraste, a las $48 \mathrm{~h}$, las ALT descendieron al $30 \%$ y a las 72 h al $56 \%$. Entre tanto, en los controles hubo un aumento de ALT en el 27,3\%, mientras que en el 15,6\% se observó una elevación de 2-9 veces, en el 7,8 \%, de 10-20 veces, y >20 veces en el 2,9\%. La combinación de cólico biliar y la elevación de ALT tuvo un valor predictivo positivo (VPP) para CDL del $72 \%$, así como un valor predictivo negativo (VPN) del 87,7\%. Conclusión: cuando hay un cólico biliar y una elevación de ALT es imperativo descartar una CDL, y si la ecografía es normal, es necesario realizar una colangiorresonancia o una ecoendoscopia biliopancreática.
\end{abstract}

\section{Palabras clave}

Coledocolitiasis, aminotransferasas, cólico biliar.

\section{Abstract}

Introduction: Choledocolithiasis (CLD) affects $10 \%$ of patients with gallstones. Bile duct obstruction is associated with pancreatitis, cholangitis, and rupture of the common bile duct. This condition usually presents with increased alkaline phosphatase, GGTP and bilirubin levels. In the last decade, it has been found that up to $10 \%$ of patients with CLD have elevated aminotransferases levels. In Latin America, this alteration has not been studied. The aim of the present work was to determine the prevalence of transaminase elevation and its evolution. Methodology: Case-control study. ALT was measured on admission, at $48 \mathrm{~h}$ and at $72 \mathrm{~h}$. If ultrasound was normal, MRCP and/or echo-endoscopy and ERCP were performed, as appropriate. Results: A total of 72 patients with choledocholithiasis (CLD) (cases) and 128 with cholecystitis without choledocholithiasis (controls) were included. Among the cases, $83 \%$ had increased ALT levels, which was 2-9 times higher in $56.9 \%, 10-20$ times higher in $16 \%$, and more than 20 times higher in $8.3 \%$. At 48 hours, those levels decreased by $30 \%$ and at 72 hours by $56 \%$. In turn, in $27.3 \%$ of the controls, ALT was $2-9$ times higher in $15.6 \%, 10-20$ times higher in $7.8 \%$ and more than 20 times higher in $2.9 \%$. The combination of biliary colic and ALT elevation had a positive predictive value (PPV) for CLD of $72 \%$ and a negative predictive value (NPV) of $87.7 \%$. Conclusion: When biliary colic and ALT elevation are reported, it is imperative to rule out choledocholithiasis. If the ultrasound is normal, MRCP and/or biliopancreatic endoscopy should be performed.

\footnotetext{
Keywords

Choledocholithiasis; Aminotransferases; Biliary colic.
} 


\section{INTRODUCCIÓN}

En la población general, la prevalencia de colelitiasis oscila entre el 5 y $10 \%$ (1). De esos pacientes, el 10-20 \% desarrollan coledocolitiasis (CDL), la cual se define como la presencia de cálculos en la vía biliar común (2). Esta enfermedad aumenta la morbimortalidad por complicaciones como pancreatitis, colangitis y ruptura del colédoco (3). Por tanto, la detección oportuna de la obstrucción de la vía biliar común es fundamental para reducir esos desenlaces (1).

Con frecuencia, el diagnóstico de CDL es difícil y exige un cuidadoso análisis clínico, bioquímico y de imágenes $(1,4)$. Muchos expertos y asociaciones científicas han estudiado y propuesto diversos criterios a fin de determinar la necesidad de realizar una colangiopancreatografía retrógrada endoscópica (CPRE) en pacientes con probable $\operatorname{CDL}(1,5,6)$. Sin embargo, este procedimiento genera complicaciones en el $15 \%$ de los pacientes (6), razón por la cual está indicado solo para propósitos terapéuticos, cuando hay un diagnóstico o una alta probabilidad de cálculos en el colédoco $(1,6)$.

En la combinación de hallazgos clínicos, de laboratorio e imágenes, la probabilidad de CDL se ha clasificado como baja $(<10 \%)$, intermedia $(10-50 \%)$ y alta $(>50 \%)(1)$. La ecografía hepatobiliar tiene una muy baja sensibilidad (22-55\%) para identificar cálculos en el colédoco, y resulta mejor al momento de determinar la dilatación del colédoco (77-87 \%) (1). Lo anterior justifica complementar el estudio con otros dos exámenes, como la colangiografía por resonancia y la ultrasonografía endoscópica, los cuales tienen una mayor sensibilidad y especificidad para identificar la $\operatorname{CDL}(1,6)$.

Recientemente, Suárez y colaboradores encontraron que, de los pacientes con probabilidad alta e intermedia de CDL, solo el 55 y el 31,4\%, respectivamente, presentaron cálculos al momento de realizar una CPRE (7). De esta manera, la sensibilidad y la especificidad de tales criterios alcanzaron el $70 \%$ (7).

Entre tanto, la mayoría de los pacientes con CDL son asintomáticos. No obstante, cuando existen síntomas, las personas que ingresan al servicio de urgencias se presentan con cólico biliar, así como con las características de dolor de aparición súbita en el epigastrio o en el hipocondrio derecho, el cual tiene una duración variable, se irradia a la espalda y está acompañado de náuseas o vómito, sin las intermitencias de un cólico usual (8).

Las alteraciones bioquímicas tradicionalmente consideradas para predecir la CDL son las que reflejan colestasis como la fosfatasa alcalina, la bilirrubina directa y la $\gamma$-glutamil-transpeptidasa (GGTP) $(1,6,8)$. Sin embargo, en las diferentes guías y recomendaciones no se ha tenido en cuenta la elevación de las aminotransferasas. En algunas series se han encontrado elevaciones marcadas de esas enzimas en pacientes con CDL (9-11). Pese a ello, en la práctica cotidiana no existen protocolos para determinar si dicha elevación de las transaminasas se debe a una enfermedad hepatocelular primaria (hepatitis aguda) o a una elevación transitoria de estas por la obstrucción del colédoco.

De esta manera, consideramos importante investigar la prevalencia y la magnitud de la elevación de las aminotransferasas, a fin de establecer si tal elevación pudiera predecir la existencia de CDL. Con ello apuntamos a estudiar profundamente a aquellos pacientes con exámenes más exactos, dado el riesgo de las complicaciones inherentes a la CDL, como la pancreatitis biliar o la colangitis, si no se resuelve la obstrucción biliar.

Además, esa hepatitis transitoria podría confundir a los clínicos y a los cirujanos, ya que si existe una elevación marcada (>10-15 veces al límite superior normal) se podría considerar, de forma errónea, que el cuadro corresponde a una hepatitis aguda, en cuyo caso se gastarían recursos innecesarios investigando la etiología de la hepatitis. Esta situación podría exponer al paciente a las complicaciones mencionadas de la obstrucción biliar por cálculos. Asimismo, operar a un paciente con hepatitis aguda tiene una alta morbilidad.

En la literatura revisada no existen trabajos prospectivos, realizados en Latinoamérica, que evalúen dicha alteración. De igual manera, tampoco hay una caracterización clínica, imagenológica y bioquímica de este grupo de pacientes que permita establecer la prevalencia de elevación de las aminotransferasas, la magnitud de la elevación y los factores asociados a ella.

Por tanto, decidimos realizar el presente trabajo con el propósito de determinar la prevalencia de hipertransaminasemia y la magnitud de esta en aquellos pacientes con CDL, así como también establecer si la elevación de esas enzimas en un individuo con cólico biliar podría predecir la CDL.

\section{MATERIALES Y MÉTODOS}

Estudio de casos y controles realizado entre noviembre de 2015 y noviembre de 2016. Se incluyeron pacientes mayores de 18 años atendidos en el servicio de urgencias de la Clínica Fundadores y el Hospital El Tunal de Bogotá (Colombia), por causa de un cuadro de cólicos biliares. Para determinar la existencia de CDL, se estudió exhaustivamente la vía biliar de todos los pacientes, mediante una ecografía hepatobiliar, una colangiografía por resonancia, una ecoendoscopia o una CPRE.

El cólico biliar se definió como un dolor de aparición súbita en el epigastrio o en el hipocondrio derecho, con una intensidad progresiva hasta alcanzar el nivel máximo, irradiarse a la espalda y acompañarse de náuseas o vómito, 
con una duración variable de minutos a horas, sin las características de los cólicos usuales (aumento y disminución de la intensidad) (8).

Dentro de ese contexto, el examen inicial fue una ecografía hepatobiliar. Cuando esta no mostró la existencia de cálculos en el colédoco o la dilatación de este $(>7 \mathrm{~mm})$, se solicitó un segundo examen más exacto, como la colangiografía por resonancia o la endosonografía biliopancreática, a fin de evaluar la vía biliar. La necesidad de realizar de manera sucesiva o aislada estos dos exámenes estuvo condicionada por la disponibilidad de dichos procedimientos. Asimismo, se utilizó la CPRE, considerado el método de referencia, cuando esta estaba indicada.

\section{Objetivo general}

Determinar la prevalencia de la elevación de aminotransferasas en los pacientes con CDL, así como el rendimiento de esta alteración como predictor de CDL.

\section{Objetivos específicos}

Caracterizar el comportamiento bioquímico de los pacientes diagnosticados con CDL e hipertransaminasemia. Además, determinar los factores predisponentes para la hipertransaminasemia en pacientes con CDL, y describir el comportamiento clínico, bioquímico y de antecedentes en aquellos pacientes con diagnóstico definitivo de CDL.

\section{Hipótesis nula}

En los pacientes con CDL no se produce una alteración de las transaminasas.

\section{Hipótesis alterna}

En los pacientes con CDL se produce una elevación de las aminotransferasas.

\section{Tamaño de la muestra}

Se incluyeron los pacientes que ingresaron con cólico biliar, a quienes les fue diagnosticado colelitiasis y CDL o CDL aislada, mediante una colangiorresonancia, una ecoendoscopia, una ultrasonografía o una CPRE con hallazgo positivo de CDL, y que, además, cumplieran con los criterios de inclusión. La muestra se ajustó al número de eventos o personas en los que se presentó una hipertransaminasemia en el rango necroinflamatorio, por el total de variables que habrían de evaluarse en el estudio. Se consideró necesario tener 10 eventos por variable incluida en el modelo.
Además, se analizaron 7 variables, razón por la cual se estimó que se requerían 70 eventos para aplicar apropiadamente un modelo de regresión logística.

\section{Criterios de inclusión}

Pacientes mayores de 18 años con diagnóstico de cólicos biliares, a quienes se les identificó litiasis biliar, mediante técnicas de imagen no invasiva, o por CPRE con hallazgo positivo para litiasis de la vía biliar común.

\section{Criterios de exclusión}

Pacientes con un cuadro clínico compatible con cólico biliar, sin litiasis biliar documentada. Pacientes con choque de cualquier etiología; hepatitis conocida de cualquier etiología; comorbilidades severas que impidan la realización de una colangiografía por resonancia, una ecoendoscopia o una CPRE; personas con diagnóstico de hepatitis aguda o crónica (autoinmune, viral, tóxica, metabólica), e individuos sin una evaluación bioquímica completa al momento de la valoración. A continuación, se describen las diferentes variables estudiadas y el protocolo de su registro (Tabla $\mathbf{1}$ ).

\section{Análisis estadístico; estadística descriptiva}

Las variables cuantitativas se evaluaron y se expresaron como promedios o medianas. Las variables cualitativas se expresaron en porcentajes o proporciones.

\section{Estadística analítica; regresión logística}

Se realizó un modelo de asociación con selección de variables por utilidad hacia adelante (forward). El determinante fundamental para incluir variables en el modelo fue el estadístico, con la prueba de Wald y la razón de verosimilitud. Se utilizó como valor de referencia una $p<0,05$ para considerar que hay significancia estadísticamente.

De igual forma, para evaluar el modelo se utilizó la prueba de bondad de ajuste de Hosmer-Lemeshow. Se realizó la identificación de eventos no típicos mediante el cálculo de: Leverages: se calculó este promedio entre los participantes y se tomaron como influenciables, o no típicos, aquellos que presentaron 3 veces o más el valor de dicho promedio.

- Residuales: se asumió que siguen una distribución chicuadrado $\left(\chi^{2}\right)$ con un grado de libertad, por lo que se interpretaron como valores influenciables aquellos $>4$.

- Influencia: se consideraron como observaciones atípicas aquellas con un valor $>0,9$. Una vez identificadas las observaciones atípicas, se evaluó la plausibilidad biológica de cada dato y se revisó si había errores de digitación. 
Tabla 1. Variables del estudio y su definición

\begin{tabular}{|c|c|c|c|c|}
\hline Variable & Definición conceptual & Definición operativa & Operatividad & $\begin{array}{l}\text { Tipo de } \\
\text { variable }\end{array}$ \\
\hline Edad & $\begin{array}{l}\text { Tiempo cronológico medido en años de } \\
\text { una persona }\end{array}$ & $\begin{array}{l}\text { Registro en la historia clínica } \\
\text { al momento del diagnóstico de } \\
\text { coledocolitiasis }\end{array}$ & Años & Continua \\
\hline Género & & Registro en la historia clínica & $\begin{array}{l}\text { 1. Masculino } \\
\text { 2. Femenino }\end{array}$ & Nominal \\
\hline Cólico biliar & $\begin{array}{l}\text { Dolor de aparición súbita en el } \\
\text { epigastrio o en el hipocondrio derecho, } \\
\text { con una intensidad progresiva hasta } \\
\text { alcanzar el nivel máximo, irradiarse a } \\
\text { la espalda y acompañarse de náuseas } \\
\text { o vómito, con una duración variable de } \\
\text { minutos a horas, sin las características } \\
\text { de los cólicos usuales (aumento y } \\
\text { disminución de la intensidad). }\end{array}$ & Registro en la historia clínica & $\begin{array}{l}\text { 1. Presente } \\
\text { 2. Ausente }\end{array}$ & Nominal \\
\hline $\begin{array}{l}\text { Duración del } \\
\text { cólico }\end{array}$ & $\begin{array}{l}\text { Tiempo en horas de dolor abdominal } \\
\text { tipo cólico biliar }\end{array}$ & $\begin{array}{l}\text { Consignación en la historia clínica } \\
\text { del tiempo de duración del dolor, } \\
\text { desde su inicio hasta el momento } \\
\text { de consulta a urgencias }\end{array}$ & Registro en horas & Continua \\
\hline Temperatura & & $\begin{array}{l}\text { Registro en la historia clínica de los } \\
\text { valores de temperatura expresados } \\
\text { en grados centígrados }\end{array}$ & $\begin{array}{l}\text { 1. Normal } \\
\text { 2. Fiebre }\left(>38,3^{\circ} \mathrm{C}\right)\end{array}$ & Ordinal \\
\hline Transaminasas & & $\begin{array}{l}\text { Registro en la historia clínica de } \\
\text { los valores de transaminasas al } \\
\text { ingreso a las } 48 \text { y } 72 \mathrm{~h} \text {. } \\
\text { Valor máximo normal en hombres: } \\
20 \text { UI/L, y en mujeres: } 20 \text { UI/L (12) }\end{array}$ & $\begin{array}{l}\text { 0. }<5 \text { veces el valor de referencia } \\
\text { 1. }<10 \text { veces el valor de referencia } \\
\text { de laboratorio } \\
\text { 2. }>10 \text { veces el valor de referencia } \\
\text { de laboratorio } \\
\text { 3. }>20 \text { veces el valor de referencia }\end{array}$ & Ordinal \\
\hline $\begin{array}{l}\text { Bilirrubina total y } \\
\text { fracciones }\end{array}$ & & $\begin{array}{l}\text { Registro en la historia clínica de la } \\
\text { bilirrubina total sérica en } \mathrm{mg} / \mathrm{dL}\end{array}$ & Registro en mg/dL & Continua \\
\hline Fosfatasa alcalina & & $\begin{array}{l}\text { Registro del valor de dicha enzima } \\
\text { en la historia clínica en UI/L al } \\
\text { ingreso, a las } 48 \text { y a las } 72 \mathrm{~h}\end{array}$ & $\begin{array}{l}\text { 0. Normal } \\
\text { 1. } 1-2 \text { veces el valor de referencia } \\
\text { de laboratorio } \\
\text { 2. > } 2 \text { veces el valor de referencia de } \\
\text { laboratorio }\end{array}$ & Ordinal \\
\hline Amilasa sérica & & $\begin{array}{l}\text { Registro en la historia clínica en } \\
\text { UI/L }\end{array}$ & $\begin{array}{l}\text { 0. }<3 \text { veces el rango de referencia } \\
\text { de laboratorio } \\
\text { 1. }>3 \text { veces el rango de referencia } \\
\text { de laboratorio }\end{array}$ & Nominal \\
\hline $\begin{array}{l}\text { Tamaño de la vía } \\
\text { biliar común }\end{array}$ & $\begin{array}{l}\text { Tamaño del colédoco, registrado } \\
\text { por ecografía, ecoendoscopia, } \\
\text { colangiorresonancia o CPRE }\end{array}$ & $\begin{array}{l}\text { Registro en la historia clínica del } \\
\text { tamaño del colédoco en mm }\end{array}$ & Registro en mm & Continua \\
\hline $\begin{array}{l}\text { Colecistectomía } \\
\text { previa }\end{array}$ & Antecedente de colecistectomía & $\begin{array}{l}\text { Registro en la historia clínica de } \\
\text { antecedente de colecistectomía }\end{array}$ & $\begin{array}{l}\text { 1. Colecistectomía previa } \\
\text { 2. Sin colecistectomía previa }\end{array}$ & Nominal \\
\hline $\begin{array}{l}\text { Índice de masa } \\
\text { corporal }\end{array}$ & $\begin{array}{l}\text { Cantidad de kilogramos por cada metro } \\
\text { de superficie corporal }\end{array}$ & $\begin{array}{l}\text { Derivado del cálculo de la relación } \\
\text { peso / talla para un momento dado }\end{array}$ & $\begin{array}{l}\text { 0. Normal: } 18-25 \mathrm{~kg} / \mathrm{m}^{2} \\
\text { 1. Sobrepeso: } 25-30 \mathrm{~kg} / \mathrm{m}^{2} \\
\text { 2. Obesidad grado I: } 30-35 \mathrm{~kg} / \mathrm{m}^{2} \text {. } \\
\text { 3. Obesidad grado II: } 35-40 \mathrm{~kg} / \mathrm{m}^{2} \\
\text { 4. Obesidad grado III }>40 \mathrm{~kg} / \mathrm{m}^{2}\end{array}$ & Ordinal \\
\hline
\end{tabular}


Tabla 1. Variables del estudio y su definición (continuación)

\begin{tabular}{|c|c|c|c|c|}
\hline Variable & Definición conceptual & Definición operativa & Operatividad & $\begin{array}{l}\text { Tipo de } \\
\text { variable }\end{array}$ \\
\hline Presión arterial & & $\begin{array}{l}\text { Antecedente de hipertensión } \\
\text { arterial crónica o uso de } \\
\text { medicación antihipertensiva }\end{array}$ & $\begin{array}{l}\text { 0. Hipertensión arterial / uso de } \\
\text { antihipertensivos } \\
\text { 1. Sin hipertensión arterial/ uso de } \\
\text { antihipertensivos }\end{array}$ & Nominal \\
\hline Diabetes mellitus & & $\begin{array}{l}\text { Antecedente de diagnóstico } \\
\text { de diabetes mellitus o uso de } \\
\text { medicación antidiabética }\end{array}$ & $\begin{array}{l}0 . \text { Presente } \\
\text { 1. Ausente }\end{array}$ & Nominal \\
\hline $\begin{array}{l}\text { Enfermedad renal } \\
\text { crónica }\end{array}$ & & $\begin{array}{l}\text { Antecedente de enfermedad renal } \\
\text { crónica }\end{array}$ & $\begin{array}{l}0 . \text { Presente } \\
\text { 1. Ausente }\end{array}$ & Nominal \\
\hline Falla cardíaca & & Antecedente de falla cardíaca & $\begin{array}{l}0 . \text { Presente } \\
\text { 1. Ausente }\end{array}$ & Nominal \\
\hline $\begin{array}{l}\text { Sustancias } \\
\text { hepatotóxicas }\end{array}$ & $\begin{array}{l}\text { Consumo de medicamentos o } \\
\text { sustancias que están definitivamente } \\
\text { relacionadas con un daño hepatocelular } \\
\text { de acuerdo con la literatura actual }\end{array}$ & $\begin{array}{l}\text { Registro en la historia clínica } \\
\text { de consumo de medicaciones } 0 \\
\text { sustancias hepatotóxicas }\end{array}$ & $\begin{array}{l}0 . \text { Presente } \\
\text { 1. Ausente }\end{array}$ & Nominal \\
\hline $\begin{array}{l}\text { Consumo de } \\
\text { alcohol }\end{array}$ & $\begin{array}{l}\text { Ingestión regular de bebidas de } \\
\text { contenido alcohólico }\end{array}$ & $\begin{array}{l}\text { Consumo de alcohol expresado } \\
\text { en g/d }\end{array}$ & $\begin{array}{l}\text { 0. }<20 \mathrm{~g} / \mathrm{d} \\
\text { 1. } 20-40 \mathrm{~g} / \mathrm{d} \\
\text { 2. }>40 \mathrm{~g} / \mathrm{d}\end{array}$ & Ordinal \\
\hline
\end{tabular}

CPRE: colangiopancreatografía retrógrada endoscópica; UI: unidades internacionales

La asociación de las variables con desenlace se expresaron mediante Odds Ratio (OR), con un intervalo de confianza (IC) del $95 \%$. El análisis de la información se hizo a través del programa STATA 13.0. StataCorp. 2013. Stata Statistical Software: Release 13. College Station, TX: StataCorp LP.

\section{Procedimientos y recolección de la información}

La información se recolectó con base en las historias clínicas de los pacientes y en el reinterrogatorio hacia ellos. Para el diagnóstico confirmado de CDL se utilizó un cuestionario (anexo). Todos los pacientes completaron el consentimiento informado. Los comités de ética de las instituciones participantes aprobaron el protocolo de investigación y dicho consentimiento informado.

\section{RESULTADOS}

En la Figura 1, se muestra el diagrama de flujo del reclutamiento de los pacientes incluidos en el estudio. Inicialmente, se evaluaron 251 pacientes con diagnóstico de litiasis biliar. Asimismo, se excluyeron 51 de ellos por diagnósticos de hepatitis viral aguda, ausencia de litiasis biliar comprobada y sospecha de hepatitis tóxica. Se incluyó un total de 200 pacientes para el análisis.

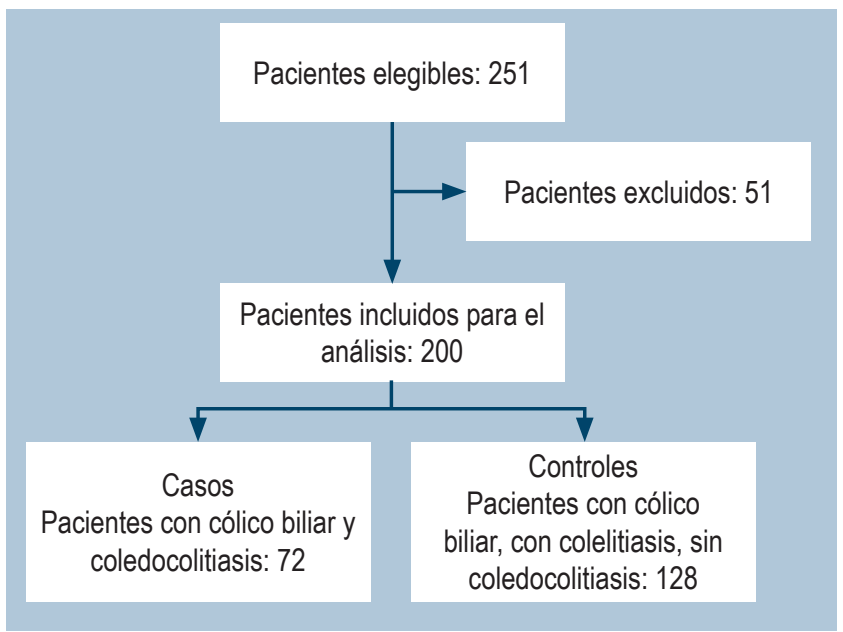

Figura 1. Diagrama de flujo de los pacientes del estudio.

El 37,5 \% (75) de los pacientes fueron hombres. Del total, $125(62,5 \%)$ cumplieron con los criterios de inclusión. Además, hubo 72 (36 \%) con CDL y 128 (64 \%) con 
colelitiasis sin CDL. Las características de los pacientes de cada grupo se muestran en la Tabla 2.

Tabla 2. Características basales de los pacientes incluidos en el estudio

\begin{tabular}{|c|c|c|c|}
\hline Variable & $\begin{array}{c}\text { Casos: } \\
\text { Cólico biliar con } \\
\text { coledocolitiasis } \\
n=72\end{array}$ & $\begin{array}{c}\text { Controles: } \\
\text { Cólico biliar sin } \\
\text { coledocolitiasis } \\
n=128\end{array}$ & $p$ \\
\hline Edad (años) & 58 & 49 & 0,014 \\
\hline Hombre (\%) & $41 \%$ & $35 \%$ & 0,83 \\
\hline AST ingreso (UI/L) & $239 \mathrm{UI} / \mathrm{L}$ & 104 UI/L & 0,016 \\
\hline ALT ingreso & $280 \mathrm{UI} / \mathrm{L}$ & $118 \mathrm{UI} / \mathrm{L}$ & 0,000 \\
\hline $\begin{array}{l}\text { Bilirrubina total de } \\
\text { ingreso }\end{array}$ & $6,8 \mathrm{mg} / \mathrm{dL}$ & $1,8 \mathrm{mg} / \mathrm{dL}$ & 0,000 \\
\hline $\begin{array}{l}\text { Bilirrubina directa al } \\
\text { ingreso }\end{array}$ & $5,6 \mathrm{mg} / \mathrm{dL}$ & $0,9 \mathrm{mg} / \mathrm{dL}$ & 0,000 \\
\hline $\begin{array}{l}\text { Fosfatasa alcalina al } \\
\text { ingreso }\end{array}$ & $420 \mathrm{UI} / \mathrm{L}$ & $171 \mathrm{UI/L}$ & 0,000 \\
\hline Amilasa sérica & $9,2 \mathrm{UI} / \mathrm{L}$ & $52,2 \mathrm{UI} / \mathrm{L}$ & 0,001 \\
\hline IMC $\left(\mathrm{kg} / \mathrm{m}^{2}\right)$ & 33,5 & 26,5 & 0,001 \\
\hline Hipertransaminasemia & $81,8 \%$ & $27,3 \%$ & 0,001 \\
\hline $\begin{array}{l}\text { Diabetes mellitus } \\
\text { tipo } 2\end{array}$ & $0 \%$ & $3,8 \%$ & \\
\hline $\begin{array}{l}\text { Enfermedad Renal } \\
\text { crónica }\end{array}$ & $1,38 \%$ & $2,3 \%$ & 0,10 \\
\hline $\begin{array}{l}\text { Consumo de } \\
\text { medicamentos }\end{array}$ & $16,6 \%$ & $29,8 \%$ & 0,004 \\
\hline Hipertensión arterial & $19 \%$ & $26 \%$ & \\
\hline
\end{tabular}

ALT: alanina aminotransferasa; AST: aspartato aminotransferasa; IMC: índice de masa corporal

De los 72 casos, 59 pacientes $(81,9 \%)$ presentaron una elevación de las aminotransferasas $\geq 2$ veces. Entre tanto, 41 participantes $(56,9 \%)$ mostraron una elevación de 2-9 veces, mientras que $12(16,6 \%)$ tuvieron una elevación de entre $10-20$ veces, y en 6 pacientes $(8,3 \%)$ se observaron elevaciones $>20$ veces (Figura 2).

Asimismo, 2 pacientes tuvieron elevaciones $>960 \mathrm{UI} / \mathrm{L}$ y 1 mostró una elevación de 1240 UI/L. De los 128 controles, 35 (27,3\%) presentaron algún grado de hipertransaminasemia $>2$ veces el límite superior normal. Además, 20 pacientes $(15,6 \%)$ presentaron elevaciones de 2-9 veces, 10 $(7,8 \%)$ tuvieron elevaciones de $10-20$ veces y en $5(3,9 \%)$ se observaron elevaciones $>20$ veces (Figura 3 ).

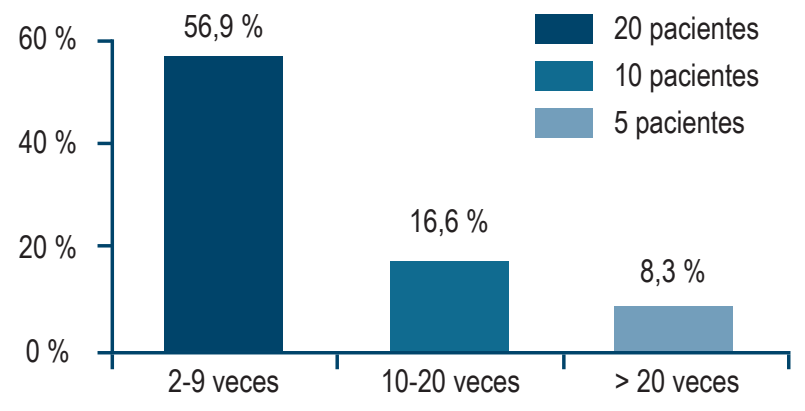

Figura 2. ALT en pacientes con CDL.

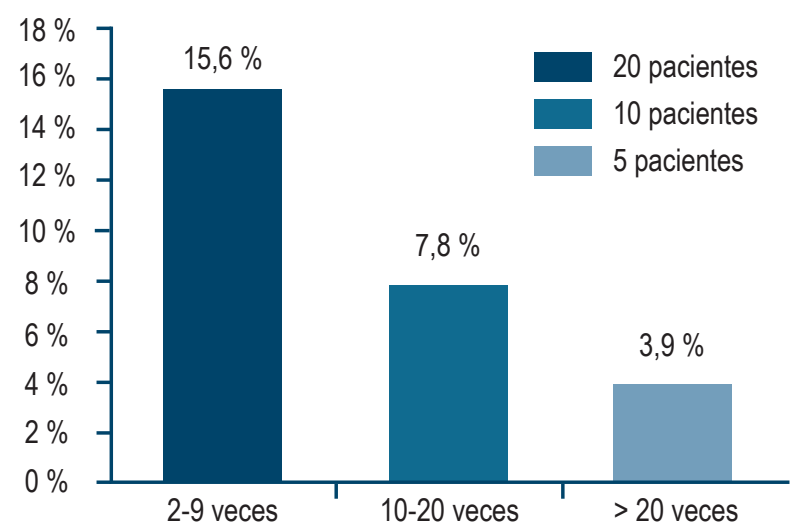

Figura 3. ALT en los pacientes sin CDL.

Entre los que tuvieron elevaciones $>20$ veces, 3 pacientes presentaron elevaciones $\geq 1000$ y 1 paciente mostró elevaciones $>2200 \mathrm{UI} / \mathrm{L}$. Los promedios de ALT y AST fueron similares, pero los de ALT resultaron un poco más altos, razón por la cual esta enzima fue tomada como la representativa (Tabla 3).

Tabla 3. Valores comparativos promedio de ALT y AST al ingreso

\begin{tabular}{|lcc|}
\hline & $\begin{array}{c}\text { Promedio } \\
\text { ingreso AST }\end{array}$ & $\begin{array}{c}\text { Promedio } \\
\text { ingreso ALT }\end{array}$ \\
\hline Mujer con coledocolitiasis & 219,52 & 289,84 \\
\hline Mujer sin coledocolitiasis & 104,007 & 110,086 \\
\hline Hombre con coledocolitiasis & 219,52 & 266,97 \\
\hline Hombre sin coledocolitiasis & 106,306 & 134,33 \\
\hline
\end{tabular}

ALT: alanina aminotransferasa; AST: aspartato aminotransferasa.

El promedio de las elevaciones de AST y ALT, así como su comportamiento, se describen a continuación. AST: al ingreso, $239 \mathrm{UI} / \mathrm{L}$; a las $48 \mathrm{~h}, 146 \mathrm{UI} / \mathrm{L}$ (descenso del $33 \%$ ); a las $72 \mathrm{~h}, 86 \mathrm{UI} / \mathrm{L}$ (descenso del $64 \%$ con respecto al valor de ingreso). Mientras que las de ALT fueron: $280 \mathrm{UI} / \mathrm{L}$, al 
ingreso; a las 48 h, $195 \mathrm{UI} / \mathrm{L}$ (descenso del $30 \%$ ) y a las 72 h, $124 \mathrm{UI} / \mathrm{L}$ (descenso del $55 \%$ con respecto al ingreso) (Figura 4).

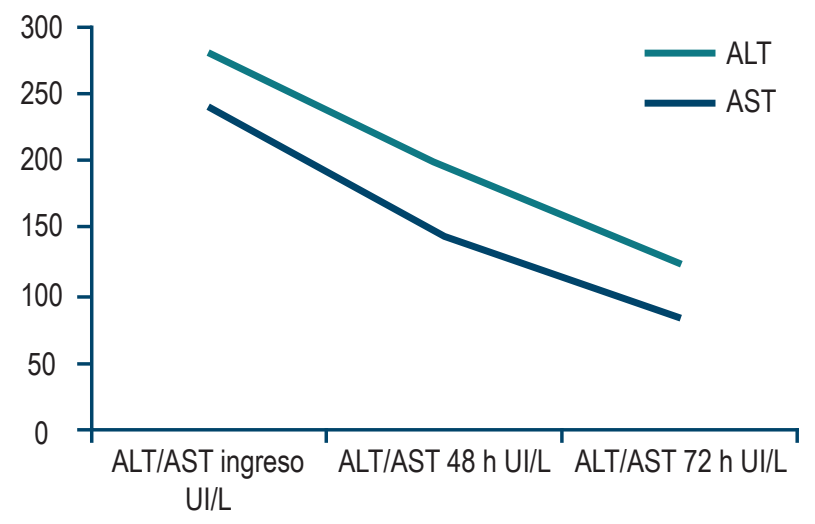

Figura 4. Comportamiento de las transaminasas en pacientes con CDL.

En el análisis multivariado, la presencia de una hipertransaminasemia confirió un OR para CDL de 4,2 (IC, $95 \%$ : $1,98-9,02)$ y, en contraste, cuando hubo CDL el riesgo de elevación de transaminasas tuvo un OR de 37,6 (IC, $95 \%$ : 1,25-112,6). A partir del análisis de regresión logística multivariada, se estableció que las variables de sexo, tiempo de duración del cólico biliar en horas, obesidad, índice de masa corporal, presencia de colecistectomía previa, falla cardíaca, enfermedad renal crónica, diabetes mellitus, consumo de alcohol y uso de medicaciones que se asocian a elevación de transaminasas no tuvieron significancia estadística para la existencia de una hipertransaminasemia en pacientes con CDL $(p>0,05)$.

De igual manera, un tamaño del colédoco $<10 \mathrm{~mm}$, la edad menor de 60 años y el número de cálculos hallados en la CPRE se asociaron positivamente con la presencia de una hipertransaminasemia en los pacientes con CDL (Tabla 4).

Tabla 4. Análisis de regresión logística multivariado para variables asociadas con la presencia o la ausencia de hipertransaminasemia en pacientes con $\mathrm{CDL}$

\begin{tabular}{|lccc|}
\hline \multicolumn{1}{|c}{ Variable } & OR & $\begin{array}{c}\text { Valor } \\
\text { de } \boldsymbol{p}\end{array}$ & IC $95 \%$ \\
\hline Edad $<60$ años & 6,5 & 0,046 & $1,03-40,98$ \\
\hline Duración del cólico & 1,003 & 0,884 & $0,955-1,05$ \\
\hline Colédoco $<10$ mm en CPRE & 18,47 & 0,036 & $1,21-281$ \\
\hline Número de cálculos encontrados en CPRE & 2,72 & 0,039 & $1,05-7,22$ \\
\hline Fiebre al momento del ingreso & 0,017 & 0,003 & $0,00122-0,252$ \\
\hline
\end{tabular}

OR: Odds Ratio; IC: intervalo de confianza; CPRE: colangiopancreatografía retrógrada endoscópica

\section{DISCUSIÓN}

Hasta donde hemos indagado, este es el primer trabajo que evalúa prospectivamente la prevalencia y el comportamiento de la elevación de las aminotransferasas en pacientes con CDL. Se encontró que el 83,3 \% de ellos tuvo un aumento de las aminotransferasas de 2 o más veces. Asimismo, el $25 \%$ de los pacientes mostró una elevación de 10 veces o más, mientras que en aquellos con colelitiasis aislada la elevación ocurrió en el 27,3 y en el 11,7 \%, respectivamente.

En este estudio se documentó que a las $48 \mathrm{~h}$ existe un descenso de más del $30 \%$ para la ALT y la AST, y a las 72 $\mathrm{h}$ el descenso de dichas enzimas fue $>50 \%$. Luego de la CPRE y la extracción de los cálculos, el descenso fue $>90 \%$. Dicho descenso continuo de las transaminasas es la clave para identificar la naturaleza transitoria de la hepatitis, cuando hay una oclusión del colédoco y un cólico biliar, y diferenciarla de una enfermedad hepatocelular primaria. La tendencia de las transaminasas hacia el descenso continuo es una clave que puede ayudar al clínico a orientar el diagnóstico de un paciente con una elevación aguda de las transaminasas y cólico biliar.

La implicación práctica de nuestros resultados sería que los pacientes con cólico biliar y las transaminasas en rango de hepatitis al momento del ingreso, que presentan un descenso significativo de las transaminasas $>30 \%$ a las $48 \mathrm{~h}, \mathrm{y}>50 \%$ a las $72 \mathrm{~h}$, no requieren estudios adicionales para hepatitis virales, tóxicas o de otra naturaleza. En lugar de ello, el clínico debe hacer un esfuerzo para establecer, a partir de métodos precisos como la ultrasonografía endoscópica y la colangiografía por resonancia, la presencia de litiasis en la vía biliar común $(7,13)$.

En dos estudios retrospectivos se identificaron pacientes con CDL y una elevación >1000 UI/L. Sin embargo, en esas investigaciones no se determinó la prevalencia de dichas elevaciones entre todos los pacientes con CDL, así como tampoco el comportamiento en el tiempo de estas $(2,14)$.

De otro lado, Suárez y colaboradores (7) identificaron a 19 pacientes de 32 con CDL, a quienes les realizaron dos determinaciones de aminotransferasas, y encontraron una elevación de ellas en el inicio del cuadro clínico, así como un descenso del $30 \%$ en la segunda determinación (7); sin embargo, estos valores no fueron comparados con los de pacientes sin CDL. Mientras tanto, en otro estudio similar, Adams y colaboradores (15) encontraron un descenso del $30 \%$ de las aminotransferasas, a partir de dos mediciones realizadas en 72 pacientes, 28 (38,9\%) de los cuales tenían CDL; el restante $61,1 \%$ no la tenían.

En esos dos estudios no se menciona si los pacientes tenían o no cólico biliar. En general, se eligieron a los participantes en virtud de su alta probabilidad de CDL, 
de acuerdo con las recomendaciones de la Sociedad Americana de Endoscopia Gastrointestinal (American Society for Gastrointestinal Endoscopy, ASGE) (1).

Durante nuestro estudio, se midieron de manera planeada las transaminasas de todos los pacientes en 3 ocasiones, sin considerar la probabilidad de realizar una preprueba, la cual es recomendada en las guías internacionales. Esta medición seriada para todos los pacientes permitió identificar un descenso del $30 \%$ a las 48 h, que alcanzó a disminuir en más del $50 \%$ a las $72 \mathrm{~h}$.

Los resultados de nuestro estudio prospectivo, y de los dos mencionados, demuestran consistentemente ese descenso del $30 \%$. Además, los hallazgos de nuestro trabajo revelaron que los pacientes con cólico biliar y elevación de las aminotransferasas tienen un OR de 4,2 (IC, $95 \%$ : $1,98-9,02)$. De forma recíproca, cuando hay CDL, la probabilidad de un aumento de las transaminasas es de 37,6. El valor predictivo positivo (VPP) de la combinación de cólico biliar con elevación de aminotransferasas (de 2 veces o más) fue del $71,9 \%$, mientras que el valor predictivo negativo (VPN) llegó al 87,7\%.

La razón de la elevación de las transaminasas en pacientes con CDL es aún desconocida. Los estudios disponibles en la actualidad han mostrado resultados variables e inconsistentes respecto a la etiología de esta enfermedad. De cualquier modo, en este estudio se evaluaron los factores relacionados con las transaminasas en rango de hepatitis como la edad, el tamaño del colédoco, el tiempo de evolución del cólico biliar, la presencia de colecistectomía previa, el sexo y las comorbilidades (diabetes, obesidad, hipertensión arterial y enfermedad renal crónica).

Dentro de ese contexto, solo la edad menor a 60 años, con un OR de 6,5, así como el tamaño del colédoco $<10$ $\mathrm{mm}$, con un OR de 18,4, y el número de cálculos encontrados en la CPRE (OR de 2,72) mostraron una asociación positiva para la presencia de hipertransaminasemia en pacientes con litiasis en la vía biliar común. La dilatación de la vía biliar es un fenómeno compensatorio para aliviar la presión dentro del colédoco. En efecto, algunos estudios han mostrado que las personas con un tamaño de la vía biliar menor tienen las transaminasas más altas $(16,17)$. Es posible que este comportamiento sea el resultado de una presión más alta dentro del árbol biliar. Los hallazgos aquí documentados son consistentes con dichas observaciones.

Por otro lado, el número de cálculos biliares encontrados en la CPRE puede relacionarse con un mayor grado de obstrucción del colédoco y con presiones más elevadas a este nivel, lo cual explicaría su asociación con la presencia de transaminasas. Múltiples estudios han encontrado que los pacientes más jóvenes tienden a presentar transaminasas en rangos más elevados, mientras numerosas referencias han develado que los enfermos de mayor edad tienen una dilatación fisiológica del colédoco. Esto puede ser un factor compensatorio que ayude a reducir la presión dentro del árbol biliar y que, además, podría explicar por qué los pacientes más jóvenes muestran un comportamiento distinto al presentar una elevación más significativa de las transaminasas ante los fenómenos obstructivos de la vía biliar común (17-19).

Los mecanismos fisiopatológicos de la elevación de aminotransferasas con la oclusión aguda del colédoco son desconocidos. No obstante, a mediados de 1970, estudios experimentales realizados en modelos animales demostraron que la ligadura del conducto biliar común en los perros producía elevaciones significativas de las transaminasas (20). De hecho, en la histología se encontró la necrosis de los hepatocitos, cuando había una obstrucción de la vía biliar común (21).

En este sentido, Mosberg (20) y Rosse (21) han descrito que la obstrucción aguda podría producir regurgitación de transaminasas desde los canalículos biliares. Entre tanto, estudios más recientes sugieren que el aumento del ácido quenodesoxicólico, generado por la colestasis aguda, induce la necrosis de los hepatocitos (22). Este proceso sería una consecuencia de la disminución del trifosfato de adenosina (Adenosine Triphosphate, ATP), del daño mitocondrial y del influjo persistente de calcio, que conducen a la muerte celular $(20,22)$.

Si bien se ha documentado que la CDL puede producir un patrón bioquímico atípico de hepatitis, sin un aumento concomitante de la fosfatasa alcalina, se desconocen las características clínicas y bioquímicas adicionales, así como el perfil de riesgos de esos pacientes. Las elevaciones de transaminasas son mayores después de la ligadura de la vía biliar extrahepática o de la inyección intravenosa de morfina en animales sometidos a una colecistectomía, en comparación con los controles $(20,21)$. La explicación propuesta para estas alteraciones es que la vesícula podría compensar las elevaciones súbitas de la presión en el tracto biliar (23).

La alta frecuencia de las elevaciones marcadas de aminotransferasas en los pacientes con colelitiasis y sin CDL, encontrada en este estudio, podría relacionarse con un mayor consumo de medicamentos hepatotóxicos en dicho grupo de pacientes, en comparación con los que presentaron CDL (29,8 \% frente al 16,6\%; $p=0,046)$. De forma alternativa, no se descarta que esos pacientes hayan tenido una migración de cálculos pequeños $(<5 \mathrm{~mm})$, a través de la vía biliar, con una resolución espontánea de la obstrucción de la vía biliar común (1).

Es posible que la no inclusión de las aminotransferasas como predictoras de CDL en las principales guías sobre el tema $(1,13)$, se deba a la falta de estudios al respecto. Consideramos que este trabajo puede contribuir al difícil manejo de estos pacientes. 


\section{Limitaciones de la investigación}

No disponer siempre del mismo equipo humano y tecnológico para evaluar la vía biliar con colangiografía por resonancia y ecoendoscopia. No se hizo un modelo utilizando variables clínicas de la CDL, las comorbilidades, el tamaño de colédoco y otros parámetros de laboratorio como la bilirrubina y la fosfatasa alcalina, a fin de predecir conjuntamente con las aminotransferasas la probabilidad de CDL. Sin embargo, el propósito de este estudio era determinar específicamente la utilidad de la elevación de las aminotransferasas como un marcador sencillo para el estudio de ese tipo de pacientes.

Otra limitación podría ser que la investigación no fue multicéntrica, aunque los estudios de esa naturaleza en nuestro medio pueden tener variables difíciles de controlar, dada la variabilidad interobservador y la no disponibilidad de expertos similares para la colangiografía por resonancia, la ecoendoscopia y la CPRE.

\section{CONCLUSIONES}

Los pacientes con cólico biliar y CDL presentan elevaciones de las aminotransferasas. Estas elevaciones son marca- das en el $25 \%$ de los pacientes y, en muchos de ellos, simulan las características de una hepatitis aguda. Sobre la base de los resultados del presente estudio, es posible afirmar que a los pacientes con cólico biliar y ecografía hepatobiliar normal se les debe solicitar un perfil hepático completo.

De igual forma, si existe un aumento de las aminotransferasas, incluidas elevaciones $>10$ veces, es necesario medirlas de manera seriada. Si se documenta un descenso significativo ( $30 \%$ a las 48 h y más del $50 \%$ a las $72 \mathrm{~h}$ ), se requiere investigar exhaustivamente la vía biliar, mediante técnicas más exactas como la colangiorresonancia o la ecoendoscopia biliopancreática, a fin de descartar la CDL.

La ausencia de una elevación de las aminotransferasas en ese tipo de pacientes tiene un VPN para CDL del 87,7 \%. Dicho en otras palabras, solo el $12,3 \%$ de individuos con cólico biliar y transaminasas normales pueden tener CDL. Este dato puede contribuir de manera sencilla al manejo de los pacientes con dicha patología.

\section{Financiación}

Los gastos de la presente investigación fueron asumidos por las instituciones participantes y por los investigadores.

\section{REFERENCIAS}

1. ASGE Standards of Practice Committee, Maple JT, BenMenachem T, Anderson MA, Appalaneni V, Banerjee S, Cash BD, Fisher L, Harrison ME, Fanelli RD, Fukami N, Ikenberry SO, Jain R, Khan K, Krinsky ML, Strohmeyer $\mathrm{L}$, Dominitz JA. The role of endoscopy in the evaluation of suspected choledocholithiasis. Gastrointest Endosc. 2010;71(1):1-9. http://doi.org/10.1016/j.gie.2009.09.041

2. Tozatti J, Mello AL, Frazon O. Predictor factors for choledocholithiasis. Arq Bras Cir Dig. 2015;28(2):109-112. http://doi.org/10.1590/S0102-67202015000200006

3. Copelan A, Kapoor BS. Choledocholithiasis: Diagnosis and Management. Tech Vasc Interv Radiol. 2015;18(4):244-255. http://doi.org/10.1053/j.tvir.2015.07.008

4. Gómez M, Pion J, Otero W. Predictores de coledocolitiasis en pacientes sometidos a colangiografía retrógrada endoscópica en el Hospital El Tunal de Bogotá. Rev Col Gastroenterol. 2011;26(4):243-252.

5. Frossard JL, Morel PM. Detection and management of bile duct stones. Gastrointest Endosc. 2010;72(4):808-816. http://doi.org/10.1016/j.gie.2010.06.033

6. ASGE Standards of Practice Committee, Anderson MA, Fisher L, Jain R, Evans JA, Appalaneni V, Ben-Menachem
T, Cash BD, Decker GA, Early DS, Fanelli RD, Fisher DA, Fukami N, Hwang JH, Ikenberry SO, Jue TL, Khan KM, Krinsky ML, Malpas PM, Maple JT, Sharaf RN, Shergill AK, Dominitz JA. Complications of ERCP. Gastrointest Endosc. 2012;75(3):467-73. http://doi.org/10.1016/j.gie.2011.07.010

7. Suárez AL, LaBarre NT, Cotton PB, Payne KM, Coté GA, Elmunzer BJ. An assessment of existing risk stratification guidelines for the evaluation of patients with suspected choledocholithiasis. Surg Endosc. 2016;30(10):4613-8. http://doi.org/10.1007/s00464-016-4799-8

8. Portincasa P, Wang DQ. Gallstones. En: Podolsky DK, Camilleri M, Fitz G, Kalloo AN, Shanahan F, Wang TC. Yamada's Textbook of Gastroenterology. Chichester: John Wiley and Sons Ltda, 6ª edición; 2016. p. 1808-1834. https://doi.org/10.1002/9781118512074.ch89

9. Bangaru S, Thiele D, Sreenarasimhaiah J, Agrawal D. Severe Elevation of Liver Tests in Choledocholithiasis: An Uncommon Occurrence With Important Clinical Implications. J Clin Gastroenterol. 2017;51(8):728-733. http://doi.org/10.1097/MCG.0000000000000608

10. Song SH, Kwon CI, Jin SM, Park HJ, Chung CW, Kwon SW, Ko KH, Hong SP. Clinical characteristics of acute 
cholecystitis with elevated liver enzymes not associated with choledocholithiasis. Eur J Gastroenterol Hepatol. 2014;26(4):452-7. http://doi.org/10.1097/MEG.0000000000000053

11. Agahi A, McNair A. Choledocholithiasis presenting with very high transaminase level. BMJ Case Rep. 2012;2012:bcr2012007268. http://doi.org/10.1136/bcr-2012-007268

12. Kim HC, Nam CM, Jee SH, Han KH, Oh DK, Suh I. Normal serum aminotransferase concentration and risk of mortality from liver diseases: prospective cohort study. BMJ. 2004;328(7446):983. http://doi.org/10.1136/bmj.38050.593634.63

13. Williams E, Beckingham I, El Sayed G, Gurusamy K, Sturgess R, Webster G, Young T. Updated guideline on the management of common bile duct stones (CBDS). Gut. 2017;66(5):765-782. http://doi.org/10.1136/gutjnl-2016-312317

14. Nathwani RA, Kumar SR, Reynolds TB, Kaplowitz N. Marked elevation in serum transaminases: an atypical presentation of choledocholithiasis. Am J Gastroenterol. 2005; 100(2):295-298. http://doi.org/10.1111/j.1572-0241.2005.40793.x

15. Adams MA, Hosmer AE, Wamsteker EJ, Anderson MA, Elta GH, Kubiliun NM, Kwon RS, Piraka CR, Scheiman JM, Waljee AK, Hussain HK, Elmunzer BJ. Predicting the likelihood of a persistent bile duct stone in patients with suspected choledocholithiasis: accuracy of existing guidelines and the impact of laboratory trends. Gastrointest Endosc. 2015;82(1):88-93. http://doi.org/10.1016/j.gie.2014.12.023

16. Hu KC, Wang HY, Chang WH, Chu CH, Lin SC, Liu CJ, Wu MS, Shih SC. Clinical presentations of patients from different age cohorts with biliary tract stone diseases. J Gastroenterol Hepatol. 2014;29(8):1614-9.

http://doi.org/10.1111/jgh.12581
17. Huh CW, Jang SI, Lim BJ, Kim HW, Kim JK, Park JS, Kim JK, Lee SJ, Lee DK. Clinicopathological features of choledocholithiasis patients with high aminotransferase levels without cholangitis: Prospective comparative study. Medicine (Baltimore). 2016;95(42):e5176. http://doi.org/10.1097/MD.0000000000005176

18. Bachar GN, Cohen M, Belenky A, Atar E, Gideon S. Effect of aging on the adult extrahepatic bile duct: a sonographic study. J Ultrasound Med. 2003;22(9):879-885. http://doi.org/10.7863/jum.2003.22.9.879

19. Barthet M, Spinoza S, Affriat C, Berthezene P, Sahel $\mathrm{J}$. Influence of age and biliary lithiasis on the diameter of the common bile duct. Gastroenterol Clin Biol. 1995;19(2):156-160.

20. Mossberg SM, Bloom A, Berkowitz J, Ross G. Serum enzyme activities following morphine. A study of transaminase and alkaline phosphatase levels in normal persons and those with gallbladder disease. Arch Intern Med. 1962;109:429-437. http://doi.org/10.1001/archinte.1962.03620160055008

21. Rosser BG, Gores GJ. Liver cell necrosis: cellular mechanisms and clinical implications. Gastroenterology. 1995;108(1):252-275. http://doi.org/10.1016/0016-5085(95)90032-2

22. Spivey JR, Bronk SF, Gores GJ. Glycochenodeoxycholateinduced lethal hepatocellular injury in rat hepatocytes. Role of ATP depletion and cytosolic free calcium. J Clin Invest. 1993;92(1):17-24. http://doi.org/10.1172/JCI116546

23. Ginsberg AL. Very high levels of SGOT and LDH in patients with extrahepatic biliary tract obstruction. Am J Dig Dis. 1970;15(9):803-807. http://doi.org/10.1007/BF02236040 